\title{
Application of an Acyl-CoA Ligase from Streptomyces aizunensis for Lactam Biosynthesis
}

Zhang, Jingwei; Barajas, Jesus F.; Burdu, Mehmet; Wang, George; Baidoo, Edward E.; Keasling, Jay D

Published in:

A C S Synthetic Biology

Link to article, DOI:

10.1021/acssynbio.6b00372

Publication date:

2017

Document Version

Publisher's PDF, also known as Version of record

Link back to DTU Orbit

Citation (APA):

Zhang, J., Barajas, J. F., Burdu, M., Wang, G., Baidoo, E. E., \& Keasling, J. D. (2017). Application of an AcylCoA Ligase from Streptomyces aizunensis for Lactam Biosynthesis. A C S Synthetic Biology, 6(5), 884-890. https://doi.org/10.1021/acssynbio.6b00372

\section{General rights}

Copyright and moral rights for the publications made accessible in the public portal are retained by the authors and/or other copyright owners and it is a condition of accessing publications that users recognise and abide by the legal requirements associated with these rights.

- Users may download and print one copy of any publication from the public portal for the purpose of private study or research.

- You may not further distribute the material or use it for any profit-making activity or commercial gain

- You may freely distribute the URL identifying the publication in the public portal 


\section{Application of an Acyl-CoA Ligase from Streptomyces aizunensis for Lactam Biosynthesis}

Jingwei Zhang, ${ }^{\#, \dagger, \S}$ Jesus F. Barajas, ${ }^{\#, \ddagger}$ Mehmet Burdu, ${ }^{\ddagger}$ George Wang, ${ }^{\ddagger}$ Edward E. Baidoo, and Jay D. Keasling $*, \dagger, \uparrow, \S, \|, \perp, \nabla, \otimes \mathbb{0}$

${ }^{\dagger}$ UCSF-UCB Joint Graduate Group in Bioengineering, University of California, Berkeley, California 94720, United States

†Joint BioEnergy Institute, Emeryville, California 94608, United States

${ }^{\S}$ Synthetic Biology Engineering Research Center, University of California, Berkeley, California 94720, United States

"Department of Chemical \& Biomolecular Engineering, University of California, Berkeley, California 94720, United States

${ }^{\perp}$ California Institute for Quantitative Biosciences, University of California, Berkeley, California 94720, United States

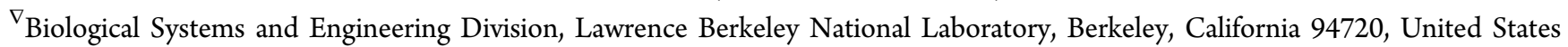

${ }^{\otimes}$ Novo Nordisk Foundation Center for Biosustainability, Technical University of Denmark, Kogle Allé, DK2970 Hørsholm, Denmark

Supporting Information

ABSTRACT: $\varepsilon$-Caprolactam and $\delta$-valerolactam are important commodity chemicals used in the manufacture of nylons, with millions of tons produced annually. Biological production of these highly valued chemicals has been limited due to a lack of enzymes that cyclize $\omega$ amino fatty acid precursors to corresponding lactams under ambient conditions. In this study, we demonstrated production of these chemicals using ORF26, an acyl-CoA ligase involved in the biosynthesis of ECO-02301 in Streptomyces aizunensis. This enzyme has a broad substrate spectrum and can cyclize 4-aminobutyric acid into $\gamma$ butyrolactam, 5-aminovaleric acid into $\delta$-valerolactam and 6-aminocaproic acid into $\varepsilon$-caprolactam. Recombinant E. coli expressing ORF26 produced valerolactam and caprolactam when 5-aminovaleric acid and 6-aminocaproic acid were added to the culture medium. Upon coexpressing ORF26 with a metabolic pathway that produced 5aminovaleric acid from lysine, we were able to demonstrate production of $\delta$-valerolactam from lysine.

$\mathrm{L}$ actams are important compounds used in the manufacture $\checkmark$ of commercial polymers. $\varepsilon$-caprolactam (caprolactam) is used in the production of nylon- 6 found in fabrics, coatings, and other plastic products. $\delta$-Valerolactam (valerolactam) has been proposed as a monomer for nylon 5 and nylon-6,5 synthesis, addition of which tunes the properties of the resulting polymers. ${ }^{1-3}$ Currently, both caprolactam and valerolactam are synthesized from petrochemical sources. For example, caprolactam production starts from cyclohexanone, which is first converted to its oxime. Treatment of this oxime with acid induces the Beckmann rearrangement to give caprolactam. ${ }^{4}$ Such production involves energy intensive processes and harsh acidic reaction conditions and produces large amount of waste salts. Unlike chemical dehydration, enzymatic or whole-cell-catalyzed reactions can be performed at lower temperatures and pressure. Although several $\omega$-amino fatty acids have been biosynthesized, ${ }^{2,5-7}$ full biosynthetic pathways to produce lactams are largely unknown. This is due to a lack of enzymes capable of performing the last ring-closing step. To date, only one enzyme, Candida antarctica lipase B (CALB, commercially available as N435), was reported to conduct a reversible aminolysis reaction that can be utilized for valerolactam and caprolactam synthesis. ${ }^{8}$ However, the reported enzyme requires anhydrous conditions, high temperatures and long reaction times. In addition, the intermolecular aminolysis reaction results in multiple side products, including macrocyclic dimer and trimer lactams, which are hard to eliminate during product purification. As such, there is no suitable enzyme capable of synthesizing industrially important lactams under microbial fermentation conditions.

Previously, ORF26 from Streptomyces aizunensis, was hypothesized to be either a 4-aminobutyryl-CoA ligase or a 4-guanidinobutyryl-CoA ligase. ${ }^{9-12}$ Previous work demonstrated that expression of ORF26 in vivo resulted in the formation of $\gamma$-butyrolactam from 4 -aminobutyric acid (4-ABA). ${ }^{13}$ In this study, we overexpressed and purified recombinant ORF26 in Escherichia coli, enabling us to explore the catalytic properties of this enzyme. ORF26 has broad substrate specificity, acting on linear or branched acid substrates with positively charged or neutral functional groups on the $\omega$ carbon. Yet the enzyme is

Received: December 9, 2016

Published: April 17, 2017 
selective against negatively charged groups on the $\omega$ carbon. ORF26's ability to activate $\omega$-amino fatty acids led to its application as a general lactam synthase, enabling biosynthesis of butyrolactam, valerolactam and caprolactam (Figure 1). Comparative study of ORF26's reaction products for different $\omega$-amino fatty acid precursors was performed to better understand this enzyme's activity as a lactam synthase.

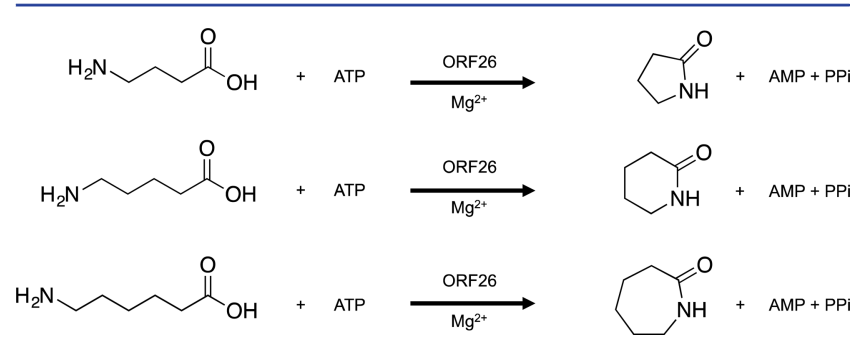

Figure 1. Proposed butyrolactam, valerolactam and caprolactam formation from their respective linear substrates catalyzed by ORF26.

To apply ORF26 for renewable chemical production, ORF26 was overexpressed in E. coli, and both valerolactam and caprolactam were formed in vivo by feeding their respective precursors, 5-aminovaleric acid (5-AVA) and 6-aminocaproic acid (6-ACA). To achieve renewable production of valerolactam, we introduced a two-enzyme pathway into E. coli that converts lysine to 5-AVA. The pathway contains an L-lysine monooxygenase and a 5-aminovaleramide amidohydrolase (davB and $\operatorname{davA}$, respectively, from Pseudomonas putida KT2440). ${ }^{1,14}$ Introducing the genes encoding the 5-AVA biosynthetic pathway and a gene encoding a fusion of ORF26 with maltose binding protein, MBP-ORF26, enabled the production of valerolactam in E. coli from lysine or directly from glucose.

\section{RESULTS}

ORF26 Enzymology. Previous efforts to express the gene encoding ORF26 in vivo resulted in the formation of $\gamma$ butyrolactam from $4-\mathrm{ABA}{ }^{13}$ In order to further explore the catalytic properties of ORF26, we overexpressed and purified recombinant ORF26 in E. coli (Table 1, Figure S1) and tested it against a range of acid substrates (Table 2) using an ATP-PP release assay. The ATP-PP ${ }_{i}$ release assay allowed us to measure ORF26's ability to activate various acid substrates with ATP. Following the ATP-PPi release assay, the samples were analyzed via LC-MS for product formation. Various apparent kinetic parameters were estimated for a total of 13 different substrates while assessing acyl-CoA or lactam formation (Table 2, Figure S2). The substrates included (1) short hydroxy- or amino-butyric acids (3-hydroxybutyric, 3-aminobutyric and 2aminobutyric acid), (2) medium-chain acids (valeric and 4methyl-hexanoic acid), (3) the $\omega$-amino fatty acids (4-ABA, 5AVA and 6-ACA), (4) $\omega$-guanidino fatty acids (4-guanidinobutyric and 6-guanidinohexanoic acid), and (5) dicarboxylic acids (glutaric, glutamic and adipic acid). In lactam formation, ORF26 demonstrated broad cyclase activity toward all three $\omega$ amino fatty acids substrates. 4-ABA, 5-AVA and 6-ACA were cyclized into butyrolactam, valerolactam and caprolactam, respectively. As an acyl-CoA ligase, ORF26 demonstrated ligase activity toward the previously hypothesized 4-guanidinobutyric acid, the longer 6-guanidinohexanoic acid and medium chain valeric and 4-methyl-hexanoic acids. ORF26 had optimal activity at $\mathrm{pH} 8.0$ (Figure S3), and therefore all in vitro reactions were conducted at $\mathrm{pH}$ 8.0. The enzyme precipitated and became inactive when the $\mathrm{pH}$ dropped below 6.0 (Figure S3).

Since ORF26 was hypothesized to be an acyl-CoA ligase, ${ }^{9-12}$ we anticipated that the ORF26-catalyzed reactions would require $\mathrm{ATP}$ or $\mathrm{ADP}$ hydrolysis to form the acyl-CoA from the acid and free CoA. As such, we monitored the in vitro reactions for both nucleotide products, in addition to the desired lactams. LC-MS analysis of nucleotides (ATP, ADP, AMP) showed that ORF26 catalyzed a series of reactions (Figure S5). In the absence of ORF26, ATP remained stable throughout the reaction (Figure S6A). In the presence of ORF26 and absence of $\omega$-amino fatty acid substrates, ATP was hydrolyzed to ADP and $\mathrm{Pi}$ and small amounts of AMP and PPi (Figure S6B). When $\omega$-amino fatty acids were added into the reaction mixture, the enzyme hydrolyzed ATP to predominantly AMP and PPi at a significantly higher rate with less formation of $\mathrm{ADP}$ and $\mathrm{Pi}$. These results suggest that the observed signal increase in the ATP-PPi assay in the presence of $\omega$-amino fatty acid substrate is due to ATP hydrolysis mainly from acid activation and a small fraction from nonspecific ATP hydrolysis by ORF26.

For lactam production, ORF26 demonstrated a higher substrate affinity for 5-AVA $\left(K_{\mathrm{m}}=0.08 \mathrm{mM}\right)$, followed by 6ACA $\left(K_{\mathrm{m}}=0.16 \mathrm{mM}\right)$ and 4-aminobutyric acid $\left(K_{\mathrm{m}}=4.96\right.$ $\mathrm{mM}$ ), using a constant concentration of ATP and CoA. Moreover, ORF26 demonstrated higher catalytic activity on 5AVA $\left(k_{\text {cat }} / K_{\mathrm{m}}=91.91 \mathrm{~min}^{-1} \mathrm{mM}^{-1}\right)$, followed by 6-ACA $\left(k_{\text {cat }}\right)$ $\left.K_{\mathrm{m}}=35.85 \mathrm{~min}^{-1} \mathrm{mM}^{-1}\right)$ and the lowest on 4-aminobutyric acid $\left(k_{\mathrm{cat}} / K_{\mathrm{m}}=1.83 \mathrm{~min}^{-1} \mathrm{mM}^{-1}\right)$. For acyl-CoA production, ORF26 demonstrated catalytic activity for both the 6guanidinohexanoic acid $\left(k_{\mathrm{cat}} / K_{\mathrm{m}}=77.83 \mathrm{~min}^{-1} \mathrm{mM}^{-1}\right)$ and 4-guanidinobutyric acid $\left(k_{\mathrm{cat}} / K_{\mathrm{m}}=18.25 \mathrm{~min}^{-1} \mathrm{mM}^{-1}\right)$ (Table 2 , Figure S2, Figure S4). In addition to acting on guanidinocontaining substrates, ORF26 also demonstrated activity toward valeric acid $\left(k_{\text {cat }} / K_{\mathrm{m}}=3.74 \mathrm{~min}^{-1} \mathrm{mM}^{-1}\right)$ and 4 -

Table 1. E. coli Strains, Plasmids Used

\begin{tabular}{cll}
\hline engineered strains & \multicolumn{1}{c}{ plasmids } \\
JZ-171 & pBbE2C-ORF26 & \multicolumn{1}{c}{ host } \\
JZ-172 & pBbE2C-RFP & JW2637-4 \\
JZ-440 & JW2637-4 \\
JZ-441 & pBbA7a-DavB-DavA & BL21(DE3) star \\
plasmid & backbone source (restriction site) & BL21(DE3) star \\
pET28b-ORF26 & pET28b (NdeI, XhoI) source (direct digestion or PCR) & pDNA2.0-ORF26 (NdeI, XhoI) \\
pET28a-MBP-ORF26 & pET28a-MBP (NdeI, XhoI) & pDNA2.0-ORF26 (NdeI, XhoI) \\
pBbA7a-DavB-DavA & pBbA7a-RFP (BglII, XhoI) & DavB, DavA gBlock \\
pET28b-CaiC & pET28b (NdeI, XhoI) & CaiC gBlock
\end{tabular}


Table 2. Steady State Parameters of ORF26 ${ }^{a, c}$

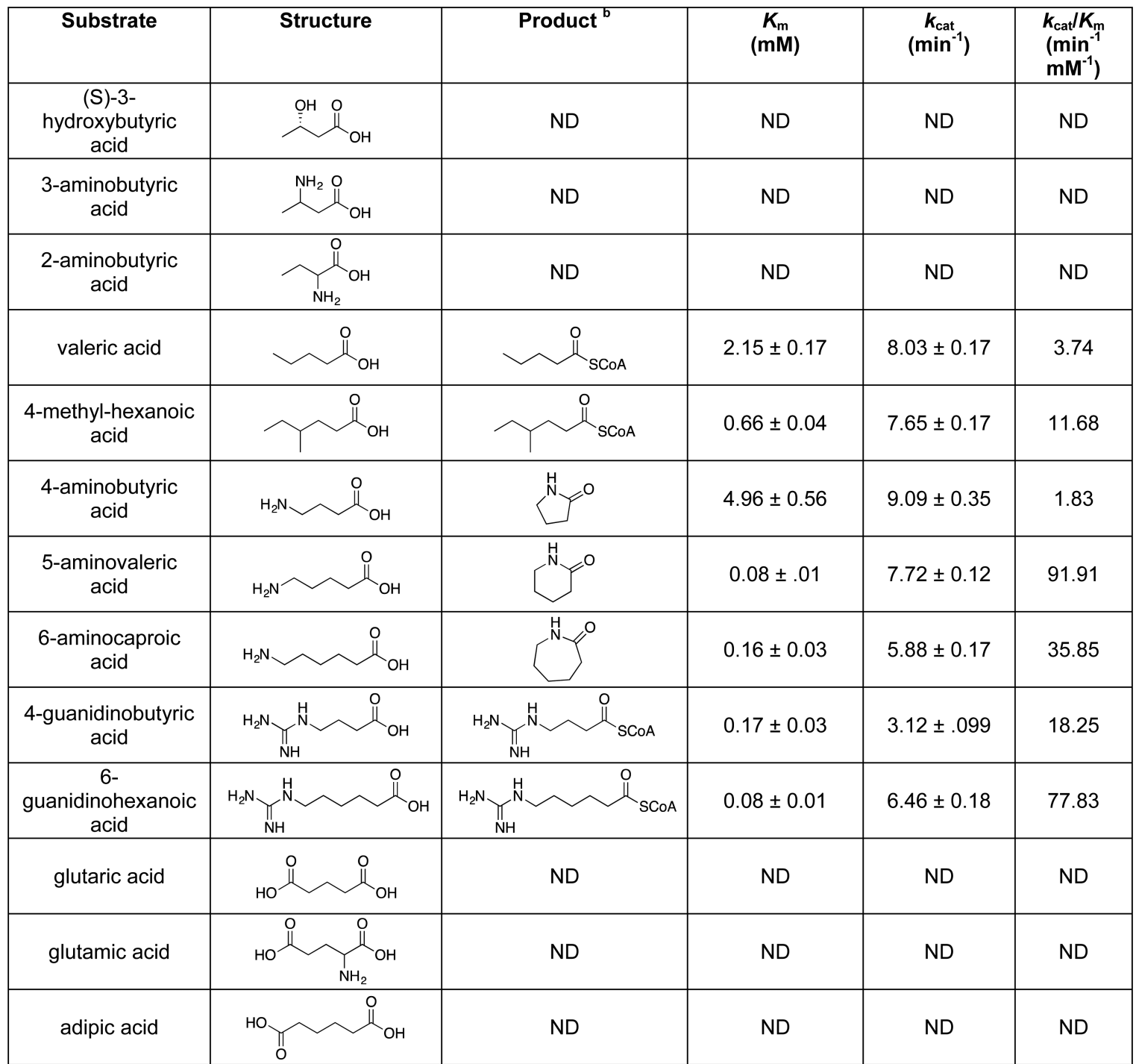

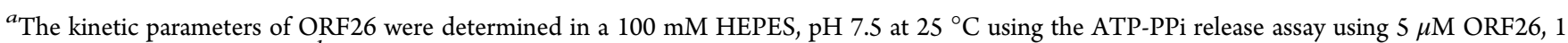
$\mathrm{mM} \mathrm{CoA}$ and $1 \mathrm{mM}$ ATP. ${ }^{b}$ Other nonproduct forming pathways could occur such as ATP hydrolysis or acyl-OAMP intermediate hydrolysis. ${ }^{c}$ Further kinetic analysis can be found in Figure S2.

methyl-hexanoic acid $\left(k_{\mathrm{cat}} / K_{\mathrm{m}}=11.68 \mathrm{~min}^{-1} \mathrm{mM}^{-1}\right)$. No activity was detected for the remaining substrates.

Although ORF26 was predicted to be an acyl-CoA ligase, CoA was not required for lactam formation (Figure S6C, E, and $\mathrm{G})$. The minimal lactam formation system consisted of ORF26, $\omega$-amino fatty acids, ATP and $\mathrm{Mg}^{2+}$ (Figure S7). Valerolactam formation was most rapid, followed by caprolactam and butyrolactam in the absence of CoA. In addition to using ATP, ORF 26 could also utilize ADP to activate $\omega$-amino fatty acids and catalyze lactam formation. The ADP reaction occurred at $\sim 20-50 \%$ of the rate compared to the ATP reaction (Figure S6I-K).

Valerolactam and Caprolactam Production In Vivo. To demonstrate valerolactam or caprolactam production using whole-cell catalysts, we exogenously added the respective precursors, $2 \mathrm{~g} / \mathrm{L}(20 \mathrm{mM})$ 5-AVA or $2 \mathrm{~g} / \mathrm{L}(17.7 \mathrm{mM}) 6$ ACA, to E. coli BL21 (DE3) star expressing pET28-ORF26 (Figure 2A,B). Both valerolactam and caprolactam were observed in the medium upon ORF26 expression (Figure $2 A, B)$. An empty pET28 vector was utilized as a control. After
$72 \mathrm{~h}$, exogenous feeding of 5-AVA and 6-ACA to the pET28ORF26 strain produced a total of $152.7 \mathrm{mg} / \mathrm{L}(1.5 \mathrm{mM})$ of valerolactam and $2.02 \mathrm{mg} / \mathrm{L}(17.9 \mu \mathrm{M})$ of caprolactam, respectively. The empty pET28 vector control displayed valerolactam production of $48.6 \mathrm{mg} / \mathrm{L}(0.5 \mathrm{mM})$ and no detectable levels of caprolactam. Valerolactam production in the empty pET28 strain suggests either a spontaneous ring closure of the linear molecule, or enzyme catalysis from endogenous host acyl-CoA enzymes. The native E. coli CaiC, a known crotonobetaine CoA ligase, was previously shown to accept 4-aminobutyric acid as substrate and catalyze the formation of butyrolactam, ${ }^{13}$ and its activity for 5-AVA and 6-ACA was also determined and compared to ORF26 (Figure 2A,B). After $72 \mathrm{~h}$, the E. coli strain expressing pET28-CaiC produced $188.8 \mathrm{mg} / \mathrm{L}(1.9 \mathrm{mM})$ of valerolactam. No detectable level of caprolactam was observed. These results suggest a broader substrate promiscuity of ORF26 as a lactam synthase in the presence of $2 \mathrm{~g} / \mathrm{L}$ lactam precursors 5-AVA and 6-ACA.

On the basis of these results, and with the aim of further investigating ORF26 in achieving renewable production of 
A

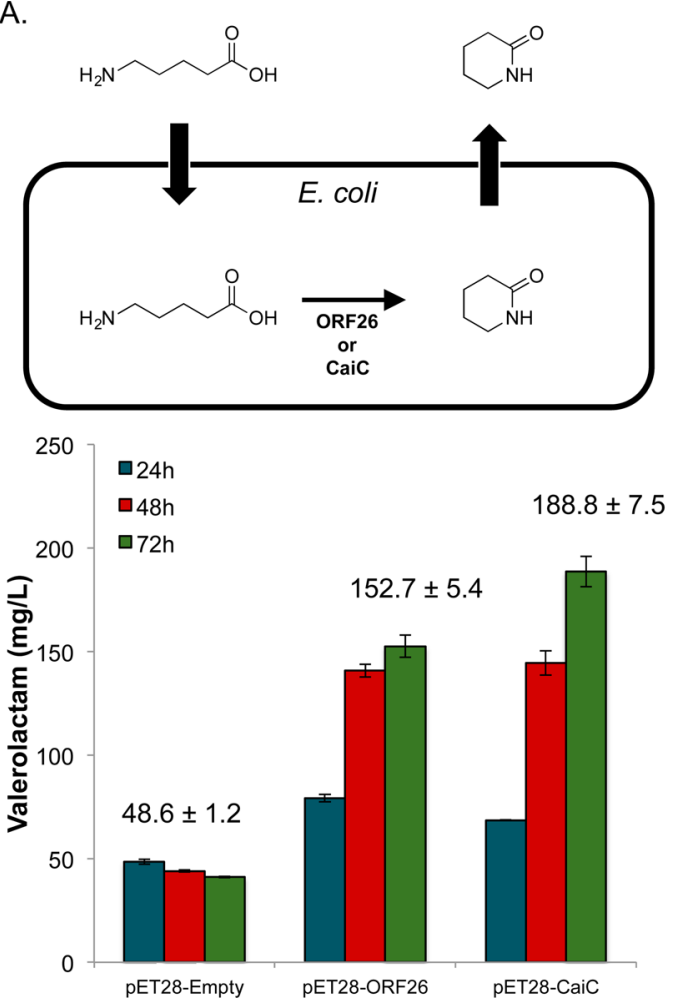

B.

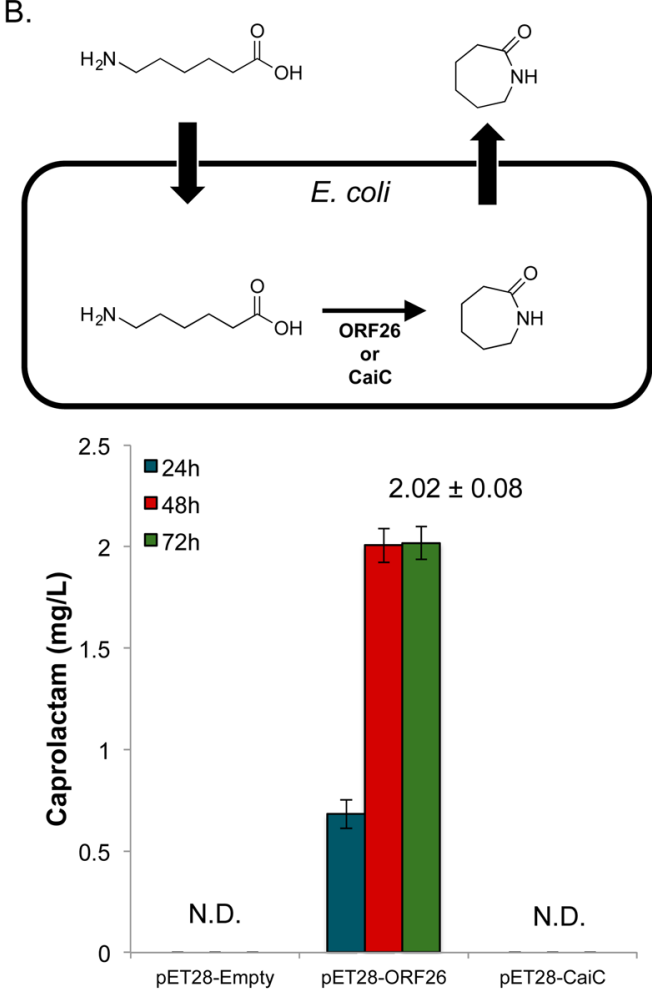

Figure 2. In vivo lactam production by ORF26 and CaiC supplemented with 5-aminovaleric acid or 6-aminocaproic acid. (A) Valerolactam biosynthesis by ORF26 and CaiC using autoinduction ZYM-5052 medium supplemented with $2 \mathrm{~g} / \mathrm{L}$ of 5-aminovaleric acid. (B) Caprolactam biosynthesis by ORF26 and CaiC using autoinduction ZYM-5052 medium supplemented with $2 \mathrm{~g} / \mathrm{L}$ of 6 -aminocaproic acid. Empty pET28 vector was used as a negative control. Not detected is denoted by N.D.

valerolactam, we introduced a two-enzyme pathway, which converts lysine to 5-AVA into E. coli BL21 (DE3) star, resulting in strain JZ-441 (Table 1). ${ }^{1-3,14}$ The pathway contains an Llysine monooxygenase and a 5-aminovaleramide amidohydrolase ( $\operatorname{dav} B$ and $\operatorname{dav} A$, respectively, from Pseudomonas putida KT2440). ${ }^{1,14}$ Introducing the genes encoding the 5-AVA biosynthetic pathway and a gene encoding a fusion of ORF26 with maltose binding protein, MBP-ORF26, enabled the production of valerolactam in E. coli from lysine (Figure 3A). JZ-440, which contains both davA and davB but lacks $M B P$ ORF26, was used as a negative control. Cultures of JZ-441 and JZ-440 were supplemented with $0,1,5$, and $10 \mathrm{~g} / \mathrm{L}$ of lysine. Samples were collected at 24,48 and 72 -h time points and analyzed via LC-MS. A linear trend was observed in valerolactam production with increasing supplemented lysine (Figure 3B). Cells growing in medium supplemented with 10 $\mathrm{g} / \mathrm{L}$ of lysine produced a maximum of $705 \mathrm{mg} / \mathrm{L}$ of valerolactam after $72 \mathrm{~h}$. At $0 \mathrm{~g} / \mathrm{L}$ lysine feeding, JZ-441 produced $146 \mathrm{mg} / \mathrm{L}$ of valerolactam after $72 \mathrm{~h}$ (Figure 3B). JZ440, which lacks MBP-ORF26, produced measurable levels of valerolactam, producing a maximum of $67 \mathrm{mg} / \mathrm{L}$ after $72 \mathrm{~h}$ when supplemented with $10 \mathrm{~g} / \mathrm{L}$ of lysine. This is most likely due to the catalysis from endogenous acyl-CoA ligase host enzymes. $^{13}$

The three-enzyme JZ-441 strain utilizes lysine, which is converted to 5-AVA by the DavB-DavA enzymatic pathway and subsequently converted into valerolactam by MBP-ORF26. In order to identify the bottleneck of this three-enzyme pathway, we measured lysine consumption and 5-AVA production in both JZ-440 and JZ-441 strains when cultured with $0 \mathrm{~g} / \mathrm{L}, 1 \mathrm{~g} /$ $\mathrm{L}(6.84 \mathrm{mM}), 5 \mathrm{~g} / \mathrm{L}(34.2 \mathrm{mM})$ and $10 \mathrm{~g} / \mathrm{L}(68.4 \mathrm{mM})$ lysine
(Figure S9). After $24 \mathrm{~h}$, both JZ-440 and JZ-441 consumed more than half of the endogenously added lysine (Figure S9A,B). By $72 \mathrm{~h}$, the majority of the supplemented lysine was converted into 5-AVA (Figure S9C,D), suggesting high stability and catalytic efficiency of both DavB and DavA in E. coli. Overall, these results suggest cyclization of 5-AVA to valerolactam by ORF26 is the rate-limiting step in this threeenzyme pathway.

\section{DISCUSSION}

Natural product biosynthesis continues to be a rich source of enzyme candidates with novel biochemical activities. ORF26, an enzyme in the Streptomyces aizunensis ECO-02301 biosynthetic cluster, was identified to be a 4-guanidinobutyryl-CoA ligase, which has interesting implications for the ECO02301 loading mechanism. ${ }^{12,15,16}$ ORF26 activated a wide range of medium chain fatty acid substrates and their functionalized analogs. Both linear and branched fatty acids were accepted as substrates. However, the enzyme seems to have little tolerance of polar groups at C3 position. ORF26 displays substrate promiscuity toward positively charged substrates, such as the $\omega$ amino fatty acids or the $\omega$-guanidino fatty acids, despite the fact that some substrates such as 6-guanidinohexanoic acid have two more carbons in their backbone than the putative native substrate. However, enzyme activity on substrates that have a negatively charged group on the $\omega$-terminal end, such as a carboxylic acid group, was not observed, even though glutaric acid and adipic acid have similar steric hindrance as 6guanidinohexanoic acid. This suggests that ORF26 has strict substrate charge specificity and preferentially favors substrates with a positively charged group on the $\omega$-terminal end. 
A.
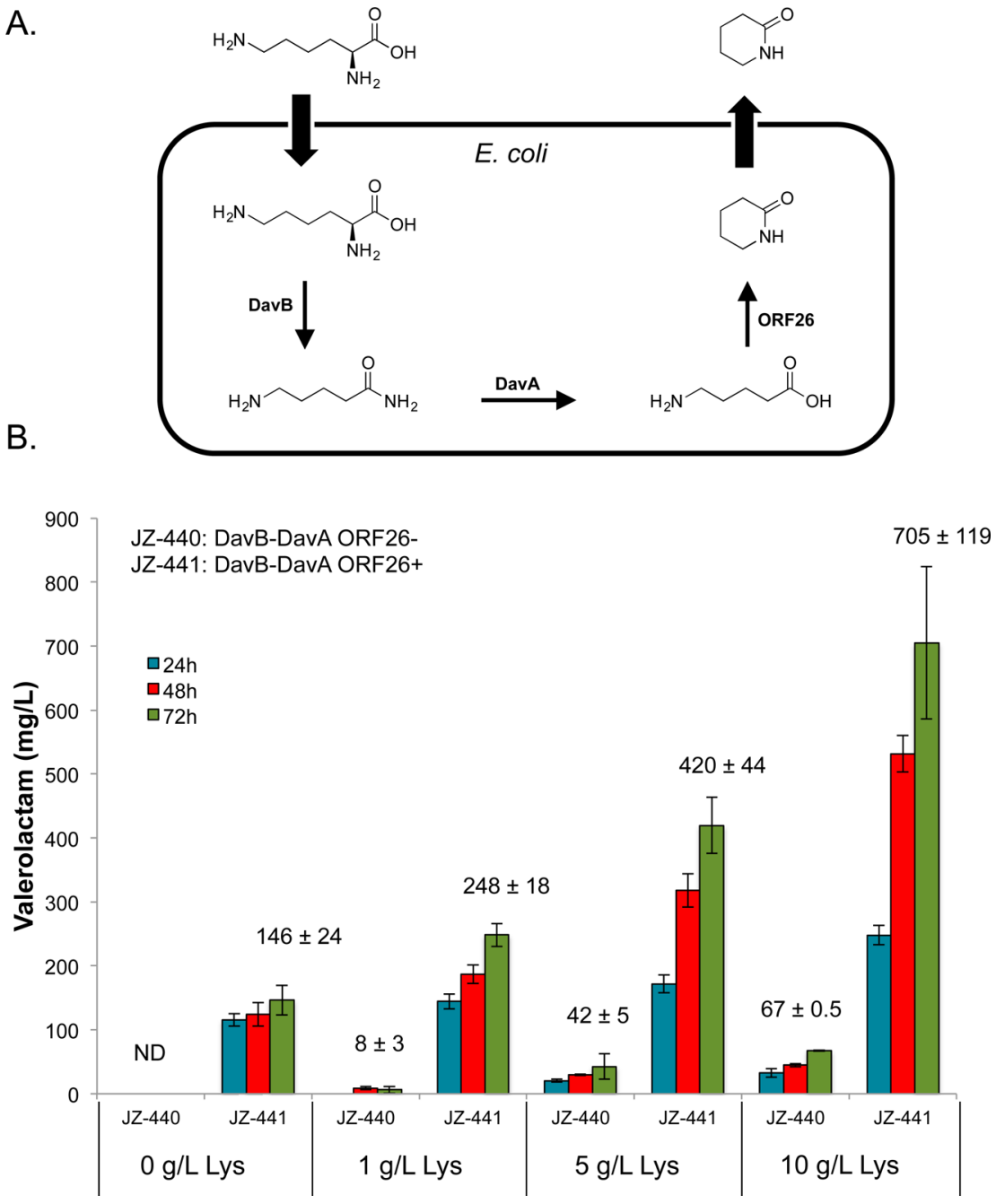

Figure 3. Valerolactam production in recombinant E. coli. (A) Valerolactam biosynthesis pathway. (B) Production of valerolactam via fermentation. Titer gain by day $1-3$ after induction in strains JZ-440 and JZ-441 from $0,1,5$ and $10 \mathrm{~g} / \mathrm{L}$ lysine feeding. JZ-440, lacking ORF26 served as a negative control. Not detected is denoted by N.D.

The ability of ORF26 to accept various $\omega$-amino fatty acids was explored for lactam biosynthesis. For lactam formation, ORF26 does not require CoA as substrate. The activation of $\omega$ amino fatty acids by ATP facilitates cyclization. This enables five-membered, six-membered and even seven-membered ring formation at mild temperatures, resulting in the production of important industrial lactams such as valerolactam and caprolactam via fermentation. Unlike the reversible aminolysis enzyme CALB that was previously described, ORF26 performs the reaction under ambient conditions. The lactam product observed is exclusively cyclized monomer, without dimer or trimer contaminants. These novel features make ORF26 an ideal candidate for lactam biosynthesis. However, the enzyme catalyzed significant ATP and ADP hydrolysis during the reaction. Directed evolution of ORF26 toward less futile ATP and ADP hydrolysis could potentially improve ORF26 as a lactam synthase.

By introducing $\operatorname{dav} B$ and $\operatorname{dav} A$ into $E$. coli, we demonstrated valerolactam biosynthesis from lysine. Valerolactam production in autoinduction medium was lysine dependent. At concentrations of lysine below $1 \mathrm{~g} / \mathrm{L}$, valerolactam production correlated with the lysine concentration from the ZYM-5052 medium. High concentrations of lysine (e.g., $10 \mathrm{~g} / \mathrm{L})$ resulted in $\sim 5$ fold increase in valerolactam production compared to nonsupplemented cultures. Even without lysine feeding, direct lysine production from endogenous lysine and glucose was sufficient to produce $\sim 50 \%$ of the valerolactam produced when $5 \mathrm{~g} / \mathrm{L}$ lysine was fed to the culture. Interestingly, in the absence of ORF26 a small amount of valerolactam was observed (Figure $3 \mathrm{~B}$, Figure S9). The observed valerolactam could result from catalysis by innate E. coli enzymes, most likely CaiC. Caprolactam was also biosynthesized by feeding its precursor 6-aminocaproic acid. With an already established 6-aminocaproic acid biosynthetic pathway in E. coli, ORF26 holds promise to enable full caprolactam biosynthesis in a microbial host. ${ }^{6,7}$

\section{CONCLUSION}

ORF26 is an enzyme in the ECO-02301 biosynthetic cluster previously identified to catalyze 4-guanidinobutyryl-CoA formation and was able to activate a wide range of substrates. While acting on $\omega$-amino fatty acids, ORF26 can catalyze the ring closing reaction to produce butyrolactam, valerolactam and caprolactam. Biosynthesis of polymer precursors such as caprolactam, valerolactam and butyrolactam were demonstrated from their respective $\omega$-amino fatty acids. E. coli harboring a 
pathway to convert lysine to 5-AVA and ORF26 were able to produce valerolactam from lysine or glucose.

\section{MATERIALS AND METHODS}

Strains and Plasmids. All the strains and plasmids utilized in this study are listed in Table 1. The link to the plasmids sequence information is listed in Supporting Information (Table S1).

ORF26 Protein Expression and Purification. The gene encoding ORF26 (GenBank: AAX98201.1) was purchased from DNA 2.0 (Menlo Park, CA). The synthetic gene was optimized with E. coli codon usage and delivered in pDNA2.0ORF26. For expression and purification of ORF26, the pDNA2.0-ORF26 plasmid was digested with NdeI and XhoI and cloned into pET28b in order to produce ORF26 with an $\mathrm{N}$-terminal 6xHis tag. The resulting plasmid, pET28b-ORF26, was transformed into E. coli BL21 Star (DE3) for ORF26 overexpression. pET28-CaiC was cloned using the same NdeI and XhoI restriction site cloning methodology as pET28ORF26.

For the N-terminal 6xHis ORF26 expression, the overnight culture was inoculated $(1: 100 \mathrm{v} / \mathrm{v})$ into $1 \mathrm{~L}$ of Luria-Bertani (LB) media containing $50 \mu \mathrm{g} / \mathrm{mL}$ kanamycin. The culture was grown at $37{ }^{\circ} \mathrm{C}$ until the $\mathrm{OD}_{600 \mathrm{~nm}}$ (optical density measured at a wavelength of $600 \mathrm{~nm}$ ) reached 0.6 and cooled on ice for 20 min. $0.5 \mathrm{mM}$ IPTG was added to induce pET28-ORF26 overexpression for $16 \mathrm{~h}$ at $18{ }^{\circ} \mathrm{C}$. The cells were harvested by centrifugation $\left(8000 \mathrm{~g}, 10 \mathrm{~min}, 4^{\circ} \mathrm{C}\right)$, resuspended in $40 \mathrm{~mL}$ of lysis buffer (50 mM HEPES, pH 8.0, $0.3 \mathrm{M} \mathrm{NaCl}, 10 \%$ glycerol (v/v) and $10 \mathrm{mM}$ imidazole), and lysed by sonication on ice. Cellular debris was removed by centrifugation $(20000 \mathrm{~g}, 60$ min, $4{ }^{\circ} \mathrm{C}$ ). The $\mathrm{N}$-terminal $6 x \mathrm{His}$ ORF26 protein was purified by ThermoFisher HisPur cobalt resin, dialyzed against $50 \mathrm{mM}$ HEPES, $\mathrm{pH}$ 8.0, $1 \mathrm{mM}$ DTT and $8 \%$ glycerol (v/v). The dialyzed proteins were concentrated to $18 \mathrm{mg} / \mathrm{mL}$ using 10 $\mathrm{kDa}$ MWCO Amicon Ultra filters (Millipore). Concentrated 6xHis ORF26 protein was aliquoted, flash frozen in liquid nitrogen and stored at $-80{ }^{\circ} \mathrm{C}$. Both DNA and protein concentrations were determined using a Nanodrop 1000 spectrophotometer (Thermo Scientific). The purified ORF26 was checked on SDS-PAGE gel for purity (Figure S1).

ATP-PPi Release Assays for ORF26. The substrate range of ORF26 was determined by ATP-PPi release assays as previously described. ${ }^{12}$ Without prior knowledge about the reaction pathways, the ATP-PPi assay served a semiquantitative method to compare substrate-induced acceleration of ATP consumption. For kinetic investigation of ORF26 activity with different substrates, the inorganic pyrophosphate released by enzymatic reaction was measured continuously using the EnzChek Pyrophosphate Assay Kit (Invitrogen). A typical assay contained in a total volume of $150 \mu \mathrm{L}: 5 \mu \mathrm{M}$ of ORF26, 0-60 mM substrates, $1 \mathrm{mM}$ ATP, $1 \mathrm{mM}$ CoA and $1 \mathrm{mM}$ $\mathrm{MgCl}_{2}$ in $100 \mathrm{mM}$ HEPES, pH 7.5. 2-amino-6-mercapto-7methylpurine ribonucleoside (MESG), purine nucleoside phosphorylase and inorganic pyrophosphatase were added according to the protocol. Reactions were initiated by the addition of ATP and monitored at $360 \mathrm{~nm}$ with SpectraMax M2 (Molecular Devices, Sunnyvale, CA). Initial velocities were calculated using the standard curve for inorganic pyrophosphate. For each substrate, control reactions were carried out without enzyme or without ATP. The rates of PPi release were converted to observed rates, and Michaelis-Menten kinetic parameters were estimated using GraphPad prism software
(GraphPad Prism version 7.00 for Mac OS X, GraphPad Software, La Jolla California USA, www.graphpad.com) (Figure S2). The acids assayed are listed in Table 2 (Sigma-Aldrich). During preparation of substrate stock solution, 6-guanidinohexanoic acid displayed low solubility under neutral $\mathrm{pH}$, and $\mathrm{HCl}$ was added to obtain $100 \mathrm{mM}$ stock solution in $\mathrm{pH} \sim 2.0$.

4-Guanidinobutyryl CoA Product Identification. To confirm ORF26's native activity as a 4-guanidinobutyryl CoA ligase, the quenched reaction with 4-guanidinobutyric acid was analyzed for CoA products as described previously. ${ }^{17}$ The mass measurements were carried out in the TOF-Scan monitoring mode for the detection of $[\mathrm{M}-\mathrm{H}]^{-}$ions (4-guanidinobutyrlCoA, $m / z=893.1825)$. Negative controls were carried out using no enzyme, no ATP, no substrate or no CoA.

Product Analysis of In Vitro ORF26 Lactam Formation. To compare formation of various lactams by ORF26, a reaction mixture containing $57 \mu \mathrm{M}$ of ORF26, $5 \mathrm{mM} \omega$-amino fatty acids substrates, $1 \mathrm{mM}$ ATP or ADP, $1 \mathrm{mM} \mathrm{CoA}$ and $1 \mathrm{mM}$ $\mathrm{MgCl}_{2}$ in $100 \mathrm{mM}$ HEPES $(\mathrm{pH}=8)$ were incubated at $25^{\circ} \mathrm{C}$. The reactions were quenched by addition of methanol to a final concentration of $50 \%(\mathrm{v} / \mathrm{v})$ at multiple time points $(0 \mathrm{~min}, 15$ min, $1 \mathrm{~h}, 2 \mathrm{~h}, 4$ and $19 \mathrm{~h}$ ). The resulting quenched reactions were kept at $4{ }^{\circ} \mathrm{C}$ and filtered through a $3 \mathrm{kDa}$ MWCO Amicon Ultra-0.5 mL Centrifugal Filters (Millipore) at $8000 \mathrm{~g}$ for 30 min. The filtered solutions were analyzed for lactams and nucleotides using HPLC-MS (see Supporting Information). Control reactions were carried out without enzyme, without substrate, without ATP, without $\mathrm{CoA}$ or without $\mathrm{MgCl}_{2}$ (supplying additional $0.2 \mathrm{mM}$ EDTA to chelate $\mathrm{Mg}^{2+}$ ions from purified protein stock). Caprolactam, valerolactam, butyrolactam, AMP, ADP and ATP were purchased as standards (Sigma-Aldrich). The $\mathrm{pH}$ dependence of ORF26catalyzed butyrolactam formation was determined using an end-point assay, and the amount of butyrolactam was determined using the HPLC-MS method described above.

In Vivo Lactam Production. E. coli JW2637-4 contains a knockout of $g a b T$, which encodes a GABA transaminase. ${ }^{18}$ This host was initially used to confirm production of valerolactam and caprolactam in vivo. E. coli JZ-171 (ORF26) and JZ-172 (RFP negative control) were inoculated overnight in LB medium, which was prepared from dehydrated powder according to the manufacturer's instructions (BD Biosciences, San Jose, CA). The overnight culture was diluted to $\mathrm{OD}_{600 \mathrm{~nm}} \sim$ 0.01 in $\mathrm{LB}$ medium and induced with $50 \mathrm{ng} / \mathrm{mL}$ anhydrotetracycline when $\mathrm{OD}_{600 \mathrm{~nm}}$ reached $\sim 0.6$. 5-AVA and 6-ACA were added exogenously into the medium to reach a final concentration of $1 \mathrm{mM}$. To analyze $24 \mathrm{~h}$ lactam production, $600 \mu \mathrm{L}$ of culture were taken for each sample and cooled in $4^{\circ}$ room. The samples were spun at $13000 \mathrm{rpm}$ for $10 \mathrm{~min}$, and $500 \mu \mathrm{L}$ of supernatant was taken and filter spin at $13000 \mathrm{rpm}$ for $10 \mathrm{~min}$ ( $0.5 \mathrm{~mL} 3000 \mathrm{MW}$ cutoff filter, EMD Millipore). $100 \mu \mathrm{L}$ filtered supernatant was taken and mixed 1:1 volume with $\mathrm{MeOH}$.

For the in vivo lactam production comparison between ORF26 and CaiC, E. coli BL21 (DE3) star cells were used as a host containing either pET28-ORF26 or pET28-CaiC. Cultures were grown in $10 \mathrm{~mL}$ of autoinduction ZYM-5052 medium according to the published protocol. ${ }^{19}$ 5-AVA and 6-ACA were added exogenously into the medium to reach a final concentration of $2 \mathrm{~g} / \mathrm{L}$. Samples were cultured at $200 \mathrm{rpm}$ and $25^{\circ} \mathrm{C}$. Samples taken at 24,48 and $72 \mathrm{~h}$ were prepared and analyzed as previously described. 
In Vivo Valerolactam Biosynthesis. The $\operatorname{dav} A$ and $\operatorname{dav} B$ genes were ordered as gBlocks (Integrated DNA Technologies, Coralville, IA) and cloned into the BglII and XhoI sites on $\mathrm{pBbA7}$, a biobrick vector, to generate plasmid pBbA7a-DavBDavA. $^{20}$ To circumvent ORF26's limited solubility during incubation, MBP-ORF26 was utilized. ${ }^{13}$ For high-density shake flask cultures, Studier's autoinduction ZYM-5052 medium was prepared according to the published protocol. ${ }^{19}$ Lysine at various concentrations $(0,1,5$ and $10 \mathrm{~g} / \mathrm{L})$ was included in the ZYM-5052 medium. Kanamycin $(20 \mu \mathrm{g} / \mathrm{mL})$ and ampicillin $(100 \mu \mathrm{g} / \mathrm{mL})$ were added where desired to provide selective pressure for plasmid maintenance.

E. coli strains (JZ-441) harboring plasmids containing $\operatorname{davA}$, davB and MBP-ORF26 were inoculated into $10 \mathrm{~mL}$ of $\mathrm{LB}$ overnight. On day 2 , the overnight culture was inoculated 1:100 (v/v) into $25 \mathrm{~mL}$ Studier's autoinduction ZYM-5052 medium with various concentrations of lysine $(0 \mathrm{~g} / \mathrm{L}$ to $10 \mathrm{~g} / \mathrm{L})$ and appropriate antibiotics. ${ }^{19}$ The culture was incubated at $37{ }^{\circ} \mathrm{C}$. When the $\mathrm{OD}_{600 \mathrm{~nm}}$ reached around 0.6 , the culture was cooled to $25{ }^{\circ} \mathrm{C}$. The culture was then placed at $25{ }^{\circ} \mathrm{C}$ incubator and the valerolactam titer was analyzed at 24,48 and $72 \mathrm{~h}$. JZ-440, which contains only $\operatorname{dav} A$ and $\operatorname{dav} B$, served as a negative control.

\section{ASSOCIATED CONTENT}

\section{S Supporting Information}

The Supporting Information is available free of charge on the ACS Publications website at DOI: 10.1021/acssynbio.6b00372.

Experimental details for ORF26 enzymology, experiments described in the main text and additional experiments (PDF)

\section{AUTHOR INFORMATION}

\section{Corresponding Author}

*Fax: + 1510495 2630. E-mail: keasling@berkeley.edu. ORCID $\odot$

Jay D. Keasling: 0000-0002-4910-2222

\section{Author Contributions}

\#.Z. and J.F.B. contributed equally.

\section{Notes}

The authors declare the following competing financial interest(s): J.D.K. has a financial interest in Amyris and Lygos.

\section{ACKNOWLEDGMENTS}

This work was funded by the Joint BioEnergy Institute (http:// www.jbei.org/), which is supported by the US Department of Energy, Office of Science, Office of Biological and Environmental Research, through contract DE-AC02-05CH11231 between Lawrence Berkeley National Laboratory and the US Department of Energy, and by the Synthetic Biology Engineering Research Center (SynBERC) through National Science Foundation grant NSF EEC 0540879. We appreciate Prof. Wenjun Zhang for discussion of the ECO-02301 biosynthesis pathway.

\section{REFERENCES}

(1) Liu, P., Zhang, H., Lv, M., Hu, M., Li, Z., Gao, C., Xu, P., and Ma, C. (2014) Enzymatic production of 5-aminovalerate from L-lysine using L-lysine monooxygenase and 5-aminovaleramide amidohydrolase. Sci. Rep. 4, 5657.

(2) Park, S. J., Kim, E. Y., Noh, W., Park, H. M., Oh, Y. H., Lee, S. H., Song, B. K., Jegal, J., and Lee, S. Y. (2013) Metabolic engineering of
Escherichia coli for the production of 5-aminovalerate and glutarate as C5 platform chemicals. Metab. Eng. 16, 42-7.

(3) Park, S. J., Oh, Y. H., Noh, W., Kim, H. Y., Shin, J. H., Lee, E. G., Lee, S., David, Y., Baylon, M. G., Song, B. K., Jegal, J., Lee, S. Y., and Lee, S. H. (2014) High-level conversion of L-lysine into 5aminovalerate that can be used for nylon 6,5 synthesis. Biotechnol. J. 9 (10), 1322-8.

(4) Josef, R. Hugo, F., Heinz, K., and William, C. M. (2005) Caprolactam. Ullmann's Encyclopedia of Industrial Chemistry, DOI: 10.1002/14356007.a05 031.pub2.

(5) Kallifidas, D., Thomas, D., Doughty, P., and Paget, M. S. (2010) The sigmaR regulon of Streptomyces coelicolor A32 reveals a key role in protein quality control during disulphide stress. Microbiology 156 (Pt 6), 1661-72.

(6) Turk, S. C., Kloosterman, W. P., Ninaber, D. K., Kolen, K. P., Knutova, J., Suir, E., Schurmann, M., Raemakers-Franken, P. C., Muller, M., de Wildeman, S. M., Raamsdonk, L. M., van der Pol, R., Wu, L., Temudo, M. F., van der Hoeven, R. A., Akeroyd, M., van der Stoel, R. E., Noorman, H. J., Bovenberg, R. A., and Trefzer, A. C. (2016) Metabolic Engineering toward Sustainable Production of Nylon-6. ACS Synth. Biol. 5 (1), 65-73.

(7) Zhou, H., Vonk, B., Roubos, J. A., Bovenberg, R. A., and Voigt, C. A. (2015) Algorithmic co-optimization of genetic constructs and growth conditions: application to 6-ACA, a potential nylon-6 precursor. Nucleic Acids Res. 43 (21), 10560-70.

(8) Stavila, E., and Loos, K. (2013) Synthesis of lactams using enzyme-catalyzed aminolysis. Tetrahedron Lett. 54 (5), 370-372.

(9) Hui Hong, T. F., and Leadlay, P. F. (2013) A Common Origin for Guanidinobutanoate Starter Units in Antifungal Natural Products. Angew. Chem. 124 (49), 13334-13337.

(10) McAlpine (2005) Microbial Genomics as a Guide to Drug Discovery and Structural Elucidation: ECO-02301, a Novel Antifungal Agent, as an Example. J. Nat. Prod. 68, 493-496.

(11) Zazopoulos (2003) A genomics-guided approach for discovering and expressing cryptic metabolic pathways. Nat. Biotechnol. 21 (2), 187-90.

(12) Zhang, A. (2010) Three Enzyme Pathway for 2-Amino-3hydroxycyclopent-2-enone Formation and Incorporation in Natural Product Biosynthesis. J. Am. Chem. Soc. 132, 6402-6411.

(13) Zhang, J., Kao, E., Wang, G., Baidoo, E. E.K., Chen, M., and Keasling, J. (2016) Metabolic Engineering of E. coli for the biosynthesis of 2-pyrrolidone. Met. Eng. Com. 3, 1.

(14) Revelles, O., Espinosa-Urgel, M., Fuhrer, T., Sauer, U., and Ramos, J. L. (2005) Multiple and interconnected pathways for L-lysine catabolism in Pseudomonas putida KT2440. J. Bacteriol. 187 (21), 7500-10.

(15) Zhang, W., Heemstra, J. R., Jr., Walsh, C. T., and Imker, H. J. (2010) Activation of the pacidamycin PacL adenylation domain by MbtH-like proteins. Biochemistry 49 (46), 9946-7.

(16) Zhang, W., and Tang, Y. (2009) In Vitro Analysis of Type II Polyketide Synthase, In Methods in Enzymology, Chapter 16, Vol. 459, pp 367-393, Elsevier.

(17) Yuzawa, S., Chiba, N., Katz, L., and Keasling, J. D. (2012) Construction of a part of a 3-hydroxypropionate cycle for heterologous polyketide biosynthesis in Escherichia coli. Biochemistry 51 (49), 9779-81.

(18) Tomoya Baba, T. A., Hasegawa, M., Takai, Y., Okumura, Y., Baba, M., Datsenko, K. A., Tomita, M., Wanner, B. L., and Mori, H. (2006) Construction of Escherichia coli K-12 in-frame, single-gene knockout mutants: the Keio collection. Mol. Syst. Biol., DOI: 10.1038/ msb4100050.

(19) Studier, W. (2005) Protein production by auto-induction in high-density shaking cultures. Protein Expression Purif. 41 (1), 207234.

(20) Lee, T. S., Krupa, R. A., Zhang, F., Hajimorad, M., Holtz, W. J., Prasad, N., Lee, S. K., and Keasling, J. D. (2011) BglBrick vectors and datasheets: A synthetic biology platform for gene expression. J. Biol. Eng. 5, 12. 